\title{
Reabilitação física na Esclerose Lateral Amiotrófica
}

\author{
Physical rehabilitation in Amyotrophic Lateral Sclerosis
}

\section{Marco Orsini ${ }^{1}$, Marcos RG de Freitas ${ }^{2}$, Mariana Pimentel Mello ${ }^{3}$, Reny deSouza Antonioli ${ }^{3}$,Jhon PetterBotelho Reis ${ }^{3}$, Osvaldo JoséMoreira Nascimento ${ }^{4}$,GabrielRodriguezdeFreitas ${ }^{5}$, CarlosHenriqueMeloReis $^{6}$}

\begin{abstract}
RESUMO
Objetivo. Atentar aos profissionais de saúde engajados com a reabilitação física, sobre os riscos existentes no tratamento de pacientes com ELA no que diz respeito ao uso excessivo ou a atrofia por desuso. Método. Foram pesquisados os artigos no período de 1958 a 2006 localizados nas bases de dados Bireme, SciELO, Pubmed, Lilacs, por meio dos seguintes descritores: escleroseamiotróficalateral, doençasneuromusculares, fadiga, fraqueza muscular, atividade física, reabilitação, fisioterapia. Resultados. O tratamento da ELA visa a prevenção da fadiga e dos danos pelo uso excessivo, além de otimizar a independência e a capacidade funcional, através de exercícios moderados, alongamentos, equipamentosdeassistênciaefisioterapia respiratória. Conclusão. Embora não haja muitos estudos de intervenção envolvendo exercícios para ganho de força em indivíduos com ELA devido ao curso variável e ao envolvimento bulbar, o fisioterapeuta, ao propor programas com tal finalidade, deve utilizar exercícios em níveis submáximos na esperança de atenuarem a perda de força, considerando a prevenção do uso excessivo e da atrofia por desuso e atentando sempre parao gerenciamento adequado destes.
\end{abstract}

Unitermos: Esclerose Amiotrófica Lateral. Doenças Neuromusculares. Fisioterapia. Reabilitação.

Citação: Orsini M, Freitas MRG de, Mello MP, Antonioli RS, Reis JPB, Nascimento OJM, Freitas GR, Reis CHM. Reabilitação física na Esclerose Lateral Amiotrófica.

Trabalho realizado no Serviço de Neurologia (Setor de

Doenças Neuromusculares) da Universidade Federal

Fluminense - UFF.

1. Graduando em Medicina, na Universidade Grande Rio - UNIGRANRIO, Doutorando em Neurociências na Universidade Federal Fluminense - UFF e Professor Titular de Reabilitação Neurológica na Escola Superior de Ensino Helena Antipoff - ESEHA.

2. Neurologista. Doutor. Professor Titular e Chefe do Serviço de Neurologia na UFF.

3. Fisioteapeutas. Centro Universitário Serra dos Órgãos - UNIFESO.

4. Neurologista, Doutor. Professor Titular de Neurologia/Neurociências e Coordenador da Pós-Graduação em Neurociências na UFF.

5. Doutor e Neurologista do Serviço de Doenças Cérebro-Vasculares na UFF.

6. Neurologista, Professor Titular da Faculdade de Medicina de Valença e Hospital da Posse.

\section{SUMMARY}

Objective. To attempt the engaged professionals of health with thephysicalrehabilitation, abouttheexisting risksinthetreatment of patients with amyotrophic lateral sclerosis (ALS) in what concerns to the extreme use or the atrophy for disuse. Method. They were researched the articles in the period of 1958 to 2006 located in the databases Bireme, SciELO, Pubmed, Lilacs, by means of thefollowingkeywords:amyotrophiclateralsclerosis,Neuromuscular disease, fatigue, muscular weakness, physical activity, rehabilitation, physiotherapy. Results. The treatment of ALS aims attheprevention of thefatigueand the damages byexcessiveuse, besides optimizing independenceand of the functional capacity, throughmoderateexercises, stretching, adaptiveequipmentsand physiotherapy respiratory. Conclusion. Although they have not manyinterventionstudiesinvolvingexercisesforimprovestrength in individuals with ALS due to the course variable and the bulbar involvement, the physical therapist, upon proposing programs with such purpose, should utilize exercises in submaximal levels inthehopeattenuatetheloss offorce,considering the prevention of the excessive use and of the atrophy for disuse and attempting always for the adequate management.

Keywords: Amyotrophic Lateral Sclerosis. Neuromuscular Diseases. Physical Therapy, Rehabilitation.

Citation: Orsini M, Freitas MRG de, Mello MP, Antonioli RS, Reis JPB, Nascimento OJM, Freitas GR, Reis CHM. Physical rehabilitation in Amyotrophic Lateral Sclerosis.

Endereço para correspondência: Marco Orsini

Rua Prof. Miguel Couto, 322/1001

24230-240 Niterói, RJ

Fone: (21) 8125-7634 e 3602-3208

E-mail: orsini@predialnet.com.br 


\section{INTRODUÇÃO}

A Esclerose Lateral Amiotrófica (ELA) é uma doença do sistemanervoso de caráter degenerativo, progressivoeincapacitante,caracterizadapelalesão dos neurônios motores no córtex, tronco cerebral e medulaespinhal,levandoaamiotrofia, fasciculações e espasticidade. Clinicamente, a doença evolui causando debilidade e atrofia progressiva da musculatura respiratória e dos membros, espasticidade, distúrbios do sono, estresse psico-social e sintomas de origem bulbar como disartria e disfagia, resultando nanecessidadedeventilaçãomecânicapermanente, e posteriormente evoluindo para o óbito. Pacientes com ELA apresentam respostas variadas ao treinamento físico, sendo essas dependentes do grau e da média de progressão da fraqueza e fadiga, além do nível de condicionamento físico. Tais atividades devem ser encaradas como uma maneira de melhorar a qualidade de vida destes, e não como a prática de exercícios extenuantes ${ }^{1-4}$.

No Brasil, em um estudo sobre as características da ELA durante o ano de 1998, foram enviados formulários estruturados a 2.505 neurologistas brasileiros para a busca de dados demográficos e clínicos dos pacientes com ELA. Quinhentos e quarenta formulários retornaram, enviados por 168 neurologistas. Foram analisados os dados de 443 pacientes que se enquadravam nos critérios de ELA provável $(14,2 \%)$ ou definida (85.8\%) de acordo com El Escorial. Duzentos e cinqüenta pacientes $(58,5 \%)$ eram do sexo masculino. A idade média de aparecimento dos primeiros sintomas foi de 52 anos. O início nos membros ocorreu em 306 pacientes (69\%), na musculatura bulbar em $82(18,5 \%)$ e a generalizada em $52(11,7 \%)$. Vinte e seis indivíduos (5,9\%) possuíam história familiar. Ajustando para a distribuição da população brasileira segundo faixa etária, a maior incidência ocorre entre 65 e 74 anos de idade. Sendo assim, a incidência desta doença a nível nacional reproduzos dados revelados no contexto mundial, no que se refere à idade e sexo ${ }^{5}$.

O tratamento da ELA conta com a participação de uma equipe multidisciplinar e inclui o tratamento farmacológico debase, otratamento sintomáticodos problemas associados eotratamento de reabilitação, com o objetivo de prolongara capacidadee independência funcional destes pacientes, visando a garantia de maior qualidade de vida possível ${ }^{6,7}$.

Este trabalho se propõe a atentar aos profissionais de saúde engajados com a reabilitação física, sobreosriscosexistentesnotratamentodepacientescom
ELA no que diz respeito ao uso excessivo ou a atrofia pordesuso, bem como conscientizá-los sobre a importância da busca do equilíbrio entre esses fatores.

\section{MÉTODO}

Trata-se de uma Revisão de Literatura baseada no que foi publicado a respeito do assunto nos últimos 50 anos, com análise descritiva. Foram pesquisados Livros e Artigos científicos (indexados) de neurologia, fisioterapia e reabilitação que abordam o tema e/ou fornecem subsídios para a elaboração destetrabalho.Osartigos científicosforam obtidosa partir de pesquisa nas bases de dados LILACS, MEDLINE, PUBMED e SciELO no período de 1958 a 2006 com os seguintes descritores: Esclerose Amiotrófica Lateral, Doenças Neuromusculares, Fadiga, Fraqueza Muscular, Fisioterapia Reabilitação.

\section{RESULTADOS}

Pacientes com doenças neuromusculares freqüentemente declaram que suas atividades físicas sãolimitadas devidoàassociação defadiga efraqueza muscular. Estes sintomas são descritos como uma exaustãoapósatividadesfísicasmínimas.Adistinção de fadiga e fraqueza é dificultada, visto que são sintomassubjetivos.Destaforma, coexistemnamaioria das DNM uma complexa relação entre fadiga muscularprimária, relacionadaàs doençasneuromusculares, e a fadiga secundária, atribuída ao descondicionamento muscular ${ }^{8}$.

A presença de fadiga crônica geralmente é relatada por pacientes com de inúmeras doenças neurológicas, tais como: Esclerose Múltipla, doenças cérebro-vasculares e Doença de Parkinson. Este sintoma pode, ainda, estar presente em indivíduos que não apresentam doenças somáticas, sendo um conceitomulti-direcionalcomcaracterísticasfisiológicas e psicológicas. Com relação ao grupo de doenças neuromusculares, a fadiga tem sido relatada por pacientes com Síndrome Pós-Pólio, Miastenia Grave, Síndrome de Guillain-Barré, ELA e nas Polineuropatias, apesardeseusmecanismoscausais(patológicosefisiológicos)aindanãoestaremtotalmente esclarecidos ${ }^{9,10}$.

Fatores como alterações metabólicas e nãometabólicas nas fibras musculares, deficiências na junção neuromuscular, mudanças biofísicas específicas nas fibras musculares e demanda metabólica intensa nas unidades motoras residuais, podem contribuir para o aparecimento da fadiga, sendo que tais mudanças podem provocar um suprimen- 
to inadequado de energia para as fibras musculares, como ocorre nos pacientes com miopatias mitocondriais ${ }^{11,12}$. Em relação a ELA a fadiga pode ser atribuídaaalteraçõesinespecíficassecundáriaadesordens não-musculares, tal como ocorre também na ParaparesiaEspástica.Emmuitospacientescomdoenças neuromusculares a fadiga central é um importante componente do processo de fadiga ${ }^{13,14}$.

Após a realização de um estudo envolvendo pacientes com poliomielite, constatou-se quea utilizaçãopermanenteeexcessivadasunidadesmotoras provocou uma transformação das fibras musculares tipo Il em fibras do tipo I, levando à hipertrofia muscular, gerando uma densidadecapilarbaixa, além de uma diminuição das atividades enzimáticas oxidativas e glicolíticas ${ }^{15}$. Tais fatores podem predispor o desenvolvimentodefraquezamuscular,fadigaanormal e dores musculares na população estudada ${ }^{16}$.

\section{DISCUSSÃO}

Para evitar a perda funcional mais rápida do que o esperado, pela história natural da doença, tanto o paciente quanto o terapeuta devem balancear delicadamente o nível de atividades entre os extremos doexercício inadequadoedoexcessivo.Doisfatores principais devem ser considerados ao planejar e implementar uma atividade ou um exercício para pacientes com ELA: prevenção da atrofia por desuso e prevenção dos danos por uso excessivo ${ }^{17}$.

Em relação a atrofia por desuso, a primeira consideraçãoaserfeitapara osterapeutasquetrabaIham com um paciente que apresenta ELA é preveni-lodedescondicionamentoeatrofiadosmúsculos, alémdonívelcausadoespecificamentepeloprocesso da doença ${ }^{17}$. Apesar de nenhum estudo ter apresentado uma relação entre a incapacidade e a atrofia pordesuso em sujeitos com diagnóstico deELA existe algumas razões pelas quais poderia ser plausível tal relação. Por ser a ELA uma doença de adultos, os pacientes podem não ter mantido suas aptidões aeróbicas ou forças musculares antes que percebam o início de seus problemas neuromusculares ${ }^{18}$.Tambémécomumaospacientesdiagnosticadosrecentementerelataremqueapresentaramumadiminuição marcante em seu nível de atividade física nos meses que precedemodiagnóstico devidoauma sensação de fadiga crescente. A fraqueza por desuso diminui a força e a resistência muscular. A atrofia por desuso em combinação com a fraqueza patológica e a espasticidadedegruposmuscularesespecíficoscontribuem para os movimentos pouco coordenados e menoseficientes, querequeremmaisgastosdeenergia, contribuindo para o nível da perda funcional e da incapacidade do paciente ${ }^{17}$.

Já no que diz respeito aos danos por uso excessivo a consideração mais crítica na elaboração de um programa de intervenção é "não causar danos". A evidência de que a atividade muscular ou a sobrecarga de exercícios pode levar à perda de força muscular foi relatada desde a epidemia de poliomielite nos anos de 1940 e $1950^{19}$.

Duranteessa epidemia, os médicose terapeutas perceberam que pacientes com músculos deficientes e de grau considerável, que realizaram exercícios repetidamente, ou com alta resistência após a reinervação, quasesempreperderamcompletamente a habilidade de contraí-los ${ }^{20}$.

Em geral, as deficiências na força muscular, a presença da espasticidade e as diminuições na amplitude de movimento estão claramente associadas às diminuições nas habilidades funcionais. Como conseqüência disso, há o interesse em relacionar as mudanças no nível de deficiência com as mudanças no nível de perda funcional. Apesar de algumas pesquisas apontarem melhorias na produção de força muscular com treinamento para o fortalecimento e a resistência dosmúsculos, osganhosfuncionaisnão foram claros ${ }^{21}$.

O fisioterapeuta deve monitorar cuidadosamente o programa de exercícios ou atividades do paciente, para assegurar que qualquer diminuição na força esteja mais relacionada com a progressão da doença do que com a sobrecarga excessiva de músculosenfraquecidos. Aodeterminaros possíveis efeitos prejudiciais das atividades, deveserfeitauma distinção entre a fadiga transitória do músculo, que muitosdenóssentimosapósum trabalhomoderado ou pesado, e a diminuição prolongada e persistente na força muscular e na resistência depois de um exercício excessivo de um músculo enfraquecido ${ }^{20}$. Se um paciente apresenta uma fraqueza significanteepersistente, posteriormenteaoestabelecimento de um programa de exercícios, ou fadiga matinal após um exercício do dia anterior, o terapeuta deve refazer o plano de exercícios e o nível de atividade, assim como aumentar a freqüência da monitoração do programa doméstico do paciente. Como os possíveis efeitos positivos e negativos dos exercícios de resistência não são claros, o terapeuta deve tomar um cuidado ainda maior com os exercícios ${ }^{17}$. Apesar deste não poder determinar o número de unidades motoras intactas disponíveis ou se o paciente está 
evocandoorecrutamentomáximodasunidadesmotoras durante as atividades, deve tomar as decisões sobre a falta ou o excesso de exercícios e ajustar o programadopaciente, baseadonarespostadesteao exercício.Oplanoterapêuticodeveserajustadoconforme a doença progride, a fim de evitar possíveis danos por falta ou excesso de atividades ${ }^{17}$.

Umpontoimportanteedegrandedificuldade naavaliaçãodestespacienteséautilizaçãodeescalas quepermitamavaliarobjetivamenteodéficitneurológico, o nível de independência funcional e, principalmente, queenquadretaisindivíduosemestágios de gravidade específicos ${ }^{22,23}$. Tais escalas podem ser específicas ou não. As específicas são: (1) a escala de avaliação funcional da ELA (ALSFRS) ${ }^{24}$; (2) o exame neuromuscular quantitativo de Tufts (TQNE) ${ }^{25}$; (3) a ALS Severity Scale (ALSSS) ${ }^{26}$; (4) a escala de Norris ${ }^{27,28}$ e (5) A ALS Health State (ALSHSS) ${ }^{29}$. Dentre as escalas não-específicas podem ser citadas: (1) a medida de independência funcional (MIF) ${ }^{30}$; (2) o índice de Barthel $^{31} ;(3)$ otestemuscularmanual(MedicalResearch Council-MRC) ${ }^{32-34}$ e(4)oMaximalVoluntarylsometric Contraction (MVIC) ${ }^{35}$.

O curso natural da ELA pode ser dividido em 6 estágios, baseados na perda progressiva da função dos músculos do tronco e das extremidades ${ }^{36}$. A identificação dos estágios do paciente pode auxiliar ofisioterapeutaa determinarumaintervençãoespecífica através do processo da doença ${ }^{37}$.

O paciente no estágio inicial da doença é independenteemrelaçãoamobilidadeeatividadesde vida diária (AVDs). Um grupo específico de músculos estálevementeenfraquecido,oquepodemanifestarseatravés delimitaçõesnodesempenhoouresistência ou ambos ${ }^{38}$. A terapia consiste em orientar o paciente e o cuidador, utilizar técnicas de conservação de energia, realizar modificações do ambiente de trabalhoe domiciliar efornecer suporte psicológico. O paciente é incentivado a continuar com as atividades físicas normais. Podem ser prescritos exercícios de amplitude de movimento ativo e alongamento global, exercícios de fortalecimento dos músculos não afetadoscom resistência moderadaeatividades aeróbicasemníveissubmáximos(natação,caminhada, bicicleta etc.) $)^{37}$.

No estágio II o paciente possui uma fraqueza moderadanosgruposmuscularesafetados, podendo apresentarmarchaescarvante,ouparesianosmúsculos intrínsecos da mão, interferindo nas habilidades motoras finas ${ }^{38}$. Um objetivo primário da intervenção nesta fase, pode ser indicar o uso de equipamen- tosdeassistência(órteses)parasuporteaosmúsculos paréticos. $\mathrm{O}$ paciente deve ser encorajado a realizar os exercícios de alongamento e amplitude de movimento ativo, fortalecimento dos músculos não afetados e atividades aeróbicas enquanto capaz. Os cuidadores e os pacientes podem ser instruídos a realizar exercícios de amplitude de movimento ativos, assistidos e passivos das articulações afetadas paraa prevenção de contraturas ${ }^{39}$.

Quando propusermos programas de fortalecimento para os pacientes com ELA nos estágios I e II, devemos considerara prevenção do uso excessivo e da atrofia por desuso ${ }^{20}$. Apesar de evidências indicarem que exercícios repetitivose/ou comalta resistência possam causar danos permanentes aos músculos enfraquecidos e desnervados ${ }^{40,41}$, uma grande redução nos níveis de atividades podem levar a um descondicionamentocardiovasculareafraquezapor desuso ${ }^{42}$.Exercíciosvigorososnãosãoindicadospara pacientes com ELA, pois para a maioria dos pacientes nenhuma atividade que não seja inerente as suas atividades diárias é indicada ${ }^{36}$. Outros autores tem relatadoefeitosbenéficosdosexercíciosdefortalecimento muscular e programas de resistência em pacientes com ELA ${ }^{21,43-45}$.

Emboraganhosfuncionaiscomoresultadosde programas de exercícios não tenham sido relatados, estudossugeremquetaisatividadespodemsersocial epsicologicamentebenéficas,especialmentequando implementados antes de haver uma grande atrofia muscular ${ }^{21,44,46-49}$. Beelo-Haas ${ }^{37}$ realizou um estudo onde foi modificado o programa de Sinak e Mulder incluindo fortalecimento muscular e exercícios de resistênciaquandotolerados, principalmentenosestágiosiniciais. Aintensidadedosexercíciosfoiajustada continuamente para prevenir a fadiga excessiva, enquantoqueaomesmotempopromoveriaummeIhordesempenhodosmúsculosintactos. Os pacientes foram advertidos a não realizar qualquer atividade ao ponto de extrema fadiga, o que resultaria em incapacidade de realizar as atividades diárias devido a exaustão, dor, fasciculações e câimbras. Alguns indivíduos com ELA apresentam câimbras e fasciculações. Os pacientes também devem ser instruídos a realizar pequenos períodos de exercícios durante o dia com tempo de repouso suficiente entre essas sessões. O tempo total de atividades recomendado durante o dia deve ser de 30 a 45 minutos, divididos em duas ou três sessões dependendo da tolerância do paciente e da resposta ao exercício ${ }^{38}$. Podem ser incluídos exercícios resistidos ativos e aeróbicos ${ }^{37,39}$. 
O paciente ainda permanece ambulatorial no estágio III, entretanto apresentando fraqueza acentuadaem determinadosgruposmusculares, quepode resultarem pécaído (marcha escarvante) egraveparesia músculos intrínsecos da mão. Tais indivíduos podem ser incapazes de se levantar de uma cadeira sem ajuda. Apesar disso apresenta uma limitação funcional de leve a moderada nas atividades quotidianas ${ }^{38}$. Nesse estágio como em todos os outros o objetivoémantera independência física efuncional do paciente. Equipamentos de assistência como órteses para tornozelo e pé, splints, cadeiras de rodas elétricas podem sernecessárias para darsuporteaos músculos fracos, diminuir o gasto energético e dar maissegurança emobilidade $\mathrm{e}^{50}$. Os pacientes podem começar a relatarfadiga e sensação de peso por conta da sustentação da cabeça. Nesse caso o uso de um colar cervical seria benéfico. Nesse estágio o uso de uma cadeira de rodas podesernecessário quandoos pacientes forem percorrer longas distâncias ${ }^{37}$.

No estágio IV o paciente apresenta uma grande fraqueza nos membros inferiores e um envolvimento leve dos membros superiores. Sendo assim com o uso de uma cadeira de rodas os pacientes podem estar aptos a realizar as suas AVDs ${ }^{38}$. Exercícios de amplitude de movimento ativo assistidose passivos são indicados para prevenir contraturas. Se ainda houverem músculos não acometidos, os exercícios deamplitude de movimentoativoe de fortalecimento devem sercontinuados. A mobilidadegeral diminui, sendo necessário a instrução em relação a inspeção da pele para as áreas de pressão. Podem se recomendadastambémdispositivosparaalternância de posição e das áreas de pressão durante o sono e enquanto o paciente estiver sentado ${ }^{39}$.

O estágio V é caracterizado por fraqueza progressiva e deterioração da mobilidade e resistência. O paciente utiliza uma cadeira de rodas quand o fora da cama, e os músculos do membro superior podem mostrar uma fraqueza moderada a severa. A transferência do paciente da cadeira de rodas exige um esforçomaiorepodesernecessária uma ajuda maior para fazê-la ${ }^{38}$. Os pacientes se tornam incapazes de semovernacama, oqueleva a necessidade decuidadosfreqüentesdereposicionamentoecuidadoscom a pele. A dor pode se tornar um problema maior nas articulações imóveis e pode requer um programa de tratamento especifico para esse sintoma. Esse programa deve ser de acordo com a fisiopatologia da dor. Um quadro álgico decorrente da espasticidade ou câimbras pode ser tratado com alongamento e massagens; dor devido a contraturas pode ser utilizadosrecursostérmicos,alongamentos, usodesplints emobilizaçãotecidual;dordevidoahipomobilidade ou traumas agudos (como quedas), costuma ser gerenciada com mobilização articular termoterapia e estimulação elétrica; dor devido a instabilidade articular pode ser tratada com uso de órteses e posicionamento adequado. Os pacientes podem ser incapazes de sustentar a cabeça por longos períodose podem necessitar do uso de um colar semi-rígido. $A$ síndrome da cabeça caída pode se iniciar nesse estágio. Se o paciente apresentar uma traqueostomia, um colar Miami-J ou um similar, que permita acesso a região anterior do pescoço liberada pode ser prescrito. A manutenção da cabeça em posição neutra irá facilitar a alimentação respiração e os cuidados como o paciente $\mathrm{e}^{37,50}$.

O paciente encontra-se restrito ao leito no estágio VI e requer assistência máxima com as atividades de vida diária. Uma cama hospitalar pode ser necessária. Mudanças de decúbito freqüentes para prevenir úlceras de pressão e também a prevençãodaestasevenosasãocuidadosfundamentais. $O$ cuidado com a dor continua sendo importante. A quedadacabeça devidoafraqueza dosmúsculosextensores do pescoço torna-se um problema maior ${ }^{38}$. Distúrbios respiratórios tornam-se progressivos e a aspiração pode ser necessária. O paciente pode necessitar defisioterapia respiratória, como mudanças de decúbito que otimizem a ventilação perfusão e a prevenção de atelectasias, drenagem postural para diminuira retenção de secreções e técnicas para mobilizar a secreção, a tosse auto-assistida (se o paciente for capaz) ou a tosse assistida. Essas técnicas são utilizadas para compensar a fraqueza dos músculos respiratórios. Devem serutilizadas técnicas paramobilizar a secreção como vibração e percussão $0^{50,51}$. Os objetivos desseestágio são similares àqueles doambiente hospitalar que seria maximizar a qualidade de vida do paciente a cada dia. A equipe multidisciplinar deve instruir os cuidadores em relação aos programasdomiciliares dependendodosproblemas edasnecessidades dos pacientes (amplitude demovimento, alongamentos, massagens, técnicas para liberação da vias aéreas, transferências) ${ }^{7,40}$.

A fraqueza dos músculos respiratórios é o principalfatorrelacionadoàmorbidadeemortalidadenasdoençasneuromusculares.Aproximadamente $90 \%$ dos episódios de falência respiratória ocorrem durante infecções de vias respiratórias superiores ${ }^{52}$. Nasinfecções respiratórias, a função pulmonaréain- 
da mais comprometida devido ao acúmulo de muco nas vias aéreas, que aumenta a resistência pulmonar e piora da disfunção dos músculos respiratórios, levando a fadiga respiratória ${ }^{53}$. A função respiratória destespacientesapresentaumpadrãorestritivo,com uma diminuição progressiva e regular da Capacidade Vital Forçada (CVF) e alteração da relação ventilação-perfusão.Oriscodecomplicaçõespulmonares aumenta conforme CVF diminui $i^{54,55}$. O objetivo da fisioterapia respiratóriaélimitara congestão pulmonar, a atelectasia e risco de falência respiratória, que poderãolevarànecessidadedeventilaçãomecânica esubseqüentetraqueostomia.Alémdafraquezados músculos respiratórios, o risco de broncoaspiraçãoe a dificuldade em eliminar secreções através da tosse contribuem para a ocorrência de infecções broncopulmonares derepetições, quepoderãoculminarem falência respiratória ${ }^{54-56}$. Treinamento dos músculos respiratórios pode auxiliar na melhora da função pulmonar, porém, deve ser aplicado de forma cautelosa para não acelerar a fadiga dos músculos respiratórios pelo aumento da sobrecarga ${ }^{57}$.

A dor pode ocorrer como resultado de problemasmusculoesqueléticosprévios (comoaosteroartrite), espasticidade, câimbras, fraqueza ou atrofia e instabilidade articular que pode causar desequilíbrios musculares e devem ser abordadas de forma apropriada no tratamento ${ }^{37}$.

\section{CONCLUSÃO}

A identificação dos estágios da doença pode auxiliar o fisioterapeuta a determinar uma intervenção específica. Tais profissionais devem ter conhecimento necessário para enquadrar o paciente no estágio correto. Embora não haja muitos estudos de intervenção envolvendo exercícios para ganho de força em indivíduos com ELA devido ao curso variável e ao envolvimento bulbar, o terapeuta, ao propor programascomtalfinalidade, deveutilizarexercícios em níveis submáximos na esperança de atenuarem a perda de força, considerando a prevenção do uso excessivoedaatrofiapordesusoeatentandosempre paraogerenciamentoadequadodestes.Infelizmente nãoexistem relatosqueapontemganhosfuncionais comessesindivíduos,logicamenteporapresentarem uma doença de caráter progressivo e degenerativo.

\section{REFERÊNCIAS BIBLIOGRÁFICAS}

1. Cassemiro $C R$, Arces CG. Comunicação visual por computador na esclerose Lateral Amiotrófica. Arq Bras Oftalmol 2004;67:295-300.
2. Voltarelli JC. Perspectivas de terapia celular na esclerose lateral amiotrófica. Rev Bras Hematol Hemoter 2004;26(3):155-6.

3. Urhán A, Herrera DA, Vargas S. Esclerosis Lateral Amiotrófica. Rev Colomb Radiol 2002;13(4):1276-9.

4. Buttarelli FR, Circella A, Pellicano C, Pontieri FE. Dopamine transporterimmunoreactivityin peripheralbloodmononuclearcellsinamyotrophic lateral sclerosis. Eur J Neurol 2006;13(4):416-8.

5. Neto FD, Callegaro D, Dias-Tosta E, Silva HA, Ferraz ME, Lima JMB, et al. Amyotrophic Lateral Sclerosis in Brazil - 1998 National Survey. Arq Neuropsiquiatr 2000; 58(3-A):607-15.

6. Calzada-Sierra DJ, Goméz-Ferenández L. Importancia del tratamiento rehabilitador multifactorial en la esclerosis lateral amiotrófica. Rev Neurol 2001;32:423-6.

7. Rocha JA, Reis C, Simoes F, Fonseca J, Mendes Ribeiro J. Diagnosticinvestigationandmultidisciplinarymanagementinmotorneuron disease. J Neurol 2005;252(12):1435-47.

8. Fowler WM. Role of physical activity and exercise training in neuromuscular diseases. Am J Phy Med Rehabil 2002;81(11 Suppl):S187-95. 9. Schulte-Mattler WJ, Muller T, Deschauer M, Gellerich FN, laizzo PA, Zierz S. Increased metabolic muscle fatigue is caused by some but not all mitochondrial muatations. Arch Neurol 2003;60:50-8.

10. Van Engelen BG, Kalkman JS, Schillings ML, Van Der Werf SP, Bleijenberg G, Zwarts MJ. Fatigue in neuromuscular disease. Ned Tijdschr Geneeskd 2004;148:1336-41.

11. Layzer RB. Muscle pain, cramps, and fatigue. In: Engel AG, FranziniAmstrong C (eds.). Myology. New York: McGraw-Hill, 1994, 1754-68.

12. Baker AJ, Carson PJ, Miller RG, Wein MW. Metabolic and nonmetabolic components of fatigue monitored with 31p-NMR. Muscle Nerve 1994; 17:1002-9.

13. Miller RG, Green AT, Moussavi RS, Carson PJ, Weiner MW. Excessive muscular fatigue in patients with spastic paraparesis. Neurology 1990;40:1271-4.

14. Sharma KR, Kent-Braun JA, Majumdar S, Huang Y, Mynhier M, Weiner MW, et al. Physiology of fatigue in amyotrophic lateral sclerosis. Neurology 1995;45:733-40.

15. Borg K, Edstrom L. Prior poliomyelitis: an immunohistochemical study of cytoskeletal proteins and a marker for muscle fibre regeneration in relation to usage of remaining motor units. Acta Neurol Scand 1993;87:128-32.

16. Borg K, Borg J, Dhoot G, Edstrom L, Grimby L, Thornell LE. Motoneuron firing and isomyosin type of muscle fibers in prior polio. $J$ Neurol Neurosurg Psychiatry 1989;52:1141-8.

17. Hallum A. Doenças neuromusculares. In: Umphred DA. Reabilitação Neurológica. 4a. ed. São Paulo: Manole, 2004, 384-440.

18. Kilmer DD, Aitkens S. Neuromuscular disease. In: Frontera WR, Dawson DM, Slovik DM. Exercise in Rehabilitation Medicine. Champaign: Human Kinetics, 1999, 253-66.

19. Russell J. Blattner Spencer WA. The treatment of acute poliomyelitis. Springfield: Charles C Thomas, 1954, 530p.

20. Bennett R L, Knowlton GC. Overwork weakness in partially denervated skeletal muscle. Clin Orthop 1958;12:22-9.

21. Sanjak M, Paulson D, Sufit R, Reddan W, Beaulieu D, Erickson L, et al. Physiologic and metabolic response to progressive and prolonged exercise in amyotrophic lateral sclerosis. Neurology 1987;37:1217-20. 22. Gomes MM. Envelhecimento e o aumento das doenças amiotróficas: epidemiologia das doenças (crônicas) das células do corno anterior da medula. Arq Bras Med 1991;65:589-94.

23. Guccione AA. Physical therapy diagnosis and the relationship between impairments and function. Phys Ther 1991;71:499-504.

24. Cedarbaum JM, Stambler N, Malta E, Fuller C, Hilt D, Thurmond $B$, et al. The ALSFRS-R: a revised ALS functional rating scale that incorporates assessments of respiratory function. BDNF ALS Study Group (Phase III). J Neurol Sci 1999;169(1-2):13-21.

25. McGuire D, Garrison L, Armon C, Barohn R, Bryan W, Miller R, et al. Relationships of the Tufts Quantitative Neuromuscular Exam (TQNE) and the Sicknes Impact Profile (SIP) in measuring progression of ALS. SSNJVCNTF ALS Study Group. Neurology 1996;46(5):1442-4.

26. Brinckmann JR, Andres P, Mendonza M, Sanjak M. Guidelines for the use and performance of quantitaive outcome measure in ALS clinical trials. J Neurol Sci 1997;147:97-111. 
27. Lacomblez L, Bensimon G, Leigh PN, Guillet P, Meininger V. Doseranging study of riluzole in amyotrophic lateral sclerosis. Amyotrophic Lateral Sclerosis/Riluzole Study Group II. Lancet 1996;347:1425-31. 28. Olarte MR, Shaffer SQ. Levamisole is ineffective in the treatment of amyotrophic lateral sclerosis. Neurology 1985;35:1063-6.

29. Desnuelle C, Dib M, Garrel C, Favier A. A double-blind, placebocontrolled randomized trial ofalfa-tocopherol(vitaminE) in thetreatment of amyotrophic lateral sclerosis. ALS riluzole-tocopherol Study Group. Amyotroph Lateral Scler Other Motor Neuron Disord 2001;2:9-18.

30. Keith RA, Granger CV, Hamilton BB, Sherwin FS. The functional independence measure: a new tool for rehabilitation. Adv Clin Rehabil 1987;1:6-18.

31. Mahoney Fl, Barthel DW. Functional evaluation: Barthel index. Md State Med J 1965;14:61-5.

32. Wrigth W. Muscle training in the treatment of infantile paralysis. Boston Med Surg J 1912;167:567.

33. Mendell J, Florence J. Manual muscle testing. Muscle Nerve 1990;13(Suppl):S16-20.

34. Brooks BR, Sufit RL, DePaul R, Tan YD, Sanjak M, Robbins J. Design of clinical therapeutic trials in amyotrophic lateral sclerosis. Adv Neurol 1991;56:521-46.

35. Beck M, Giess R, Wurffel W, Magnus T, Ochs G, Toyka KV. Comparison of maximal voluntary isometric contraction and Drachman's hand-helddynamometryinevaluating patientswithamyotrophiclateral sclerosis. Muscle Nerve 1999;22:1265-70.

36. Sinaki M. Rehabilitation. In: Mulder DW. The Diagnosis and Treatment of Amyotrophic Lateral Sclerosis. Boston: Houghton Mifflin Co, 1980, 171-93. 37. Dal Beelo-Haas V, Kloos AD, Mitsumoto H. Physical Therapy for a Patient Through Six Stages of Amyotrophic Lateral Sclerosis. Phys Ther 1998;78(12):1312-24.

38. Sinaki M, Mulder DW. Rehabilitation techniques for patients with amyotrophic lateral sclerosis. Mayo Clin Proc 1978;53:173-8.

39. Orient-López F, Terré-Boliart R, Guevara-Espinosa D, BernabeuGuitart. Tratamiento neurorrehabilitador de la esclerosis lateral amiotrófica. Rev Neurol 2006;43(9):549-55.

40. Smith RA, Norris FH. Symptomatic care of patients with amyotrophic lateral sclerosis. JAMA 1975;234:715-7.

41. Wagner MB, Vignos PJ, Fonow DC . Serial isokinetic evaluations used for a patient with scapuloperoneal muscular dystrophy: a case report. Phys Ther 1986;66:1110-3.

42. Norris FH. Exercise for patients with neuromuscular diseases. West J Med 1985;142:261.

43. Bohannon RW. Results of resistance exercise on a patient with amyotrophic lateral sclerosis: a case report. Phys Ther 1983;63:965-8.
44. Kilmer DD, McCrory MA, Wright NC, Aitkens SG, Bernauer EM. The effect of a high resistance exercise program in slowly progressive neuromuscular disease. Arch Phys Med Rehabil 1994;75:560-3.

45. Drory VE, Goltsman E, Reznik JG, Mosek A, Korczyn AD. The value of muscle exercise in patients with amyotrophic lateral sclerosis. J Neurol Sci 2001;191(1-2):133-7.

46. Aitkens SG, McCrory MA, Kilmer DD, Bernauer EM. Moderate resistanceexerciseprogram:its effectinslowlyprogressiveneuromuscular disease.Arch Phys Med Rehabil 1993;74(7):711-5.

47. Milner-Brown HS, Miller RG. Muscle strengthening through electric stimulation combined with low-resistance weights in patients with neuromuscular disorders. Arch Phys Med Rehabil 1988;69:20-4.

48. Wright NC, Kilmer DD, McCrory MA, Aitkens SG, Holcomb BJ, Bernauer EM. Aerobic walking in slowly progressive neuromuscular disease: effect of a 12-week program. Arch Phys Med Rehabil 1996;77:64-9. 49. Florence JM, Hagberg JM. Effect of training on the exercise responses of neuromuscular disease patients. Med Sci Sports Exerc 1984;16:460-5.

50. Desnuelle C, Bruno M, Soriani MH, Perrin C. Quelles sont les modalités dethérapiephysiquesymptomatiqueincluantlestechniques de désencombrement bronchique? Rev Neurol (Paris) 2006;162(Spec 2):4S244-52.

51. Frownfelter D, Dean E. Principle and Practice of Cardiopulmonary Physical Therapy. 3rd ed. St Louis: CV Mosby Co, 1996, 435p.

52. Bach JR, Rajaraman R, Ballanger F, 6 autores, et al. Neuromuscularventilatoryinsufficiency:theeffectofhomemechanicalventilatoruse vs oxygen therapy on pneumonia and hospitalization rates. Am J Phys Med Rehabil 1998;77:8-19.

53. Tzeng AC, Bach JR. Prevention of pulmonary morbidity for patients with neuromuscular disease. Chest 2000;118(5):1390-6.

54. Ioos C, Leclair-Richard D, Mrad S, Barois A, Estournet-Mathiaud B. Respiratory capacity course in patients with infantile spinal muscular atrophy. Chest 2004;126(3):831-7.

55. Tangsrud SE, Carlsen KC, Lund-Petersen I, Carlsen KH. Lung function measurements in young children with spinal muscle atrophy; a cross sectional survey on the effect of position and bracing. Arch Dis Child 2001;84(6):521-4.

56. Miske LJ, Hickey EM, Kolb SM, Weiner DJ, Panitch HB. Use of the mechanical in-exsufflator in pediatric patients with neuromuscular disease and impaired cough. Chest 2004;125(4):1406-12.

57. Koessler W, Wanke T, Winkler G, Nader A, Toifl K, Kurz H, et al. 2 Years' experience with inspiratory muscle training in patients with neuromuscular disorders. Chest 2001;120(3):765-9. 